Recherches en didactique des langues et des cultures

Les cahiers de l'Acedle

\title{
L'accompagnement professionnel pour développer des dispositifs didactiques innovants en syntaxe et en ponctuation : regards de conseillères pédagogiques
}

Marie-Hélène Giguère, Marie Nadeau, Carole Fisher, Rosianne Arseneau et Claude Quevillon Lacasse

\section{OpenEdition}

\section{Journals}

Édition électronique

URL : https://journals.openedition.org/rdlc/7457

DOI : $10.4000 /$ rdlc. 7457

ISSN : 1958-5772

Éditeur

ACEDLE

Référence électronique

Marie-Hélène Giguère, Marie Nadeau, Carole Fisher, Rosianne Arseneau et Claude Quevillon Lacasse, "L'accompagnement professionnel pour développer des dispositifs didactiques innovants en syntaxe et en ponctuation : regards de conseillères pédagogiques ", Recherches en didactique des langues et des cultures [En ligne], 17-2 | 2020, mis en ligne le 27 avril 2020, consulté le 17 octobre 2022. URL : http://journals.openedition.org/rdlc/7457 ; DOI : https://doi.org/10.4000/rdlc.7457

Ce document a été généré automatiquement le 17 octobre 2022. 


\section{L'accompagnement professionnel pour développer des dispositifs didactiques innovants en syntaxe et en ponctuation : regards de conseillères pédagogiques}

Marie-Hélène Giguère, Marie Nadeau, Carole Fisher, Rosianne Arseneau et Claude Quevillon Lacasse

\section{Introduction}

1 Depuis plusieurs années, des recherches européennes et québécoises portant sur les pratiques effectives des enseignants en syntaxe et en ponctuation (S-P) montrent que cet enseignement est isolé et que les exercices proposés n'engagent pas les élèves à construire des savoirs suffisamment solides pour leur permettre de réaliser un transfert en situation d'écriture (Chartrand, 2009; Jaffré, 2014; Paolacci et GarciaDebanc, 2003; Paolacci et Rossi-Gensane, 2014). Or, les recherches didactiques sur l'enseignement et l'apprentissage de l'orthographe grammaticale (Brissaud et Cogis, 2011 ; Nadeau et Fisher, 2014) fournissent des pistes encourageantes pour améliorer les compétences des élèves en écriture puisque la résolution de problèmes orthographiques en situation de rédaction passe largement par la mobilisation et l'utilisation explicite des connaissances grammaticales et syntaxiques (Boivin et Pinsonneault, 2014 ; Nadeau et Fisher, 2009, 2011).

2 C'est dans ce contexte qu'une recherche quasi-expérimentale ${ }^{1}$ a vu le jour pour tenter d'observer les effets de la mise en œuvre de différents dispositifs didactiques jugés prometteurs pour enseigner la S-P dans des classes du primaire et du secondaire québécois. Or, l'adaptation de ces dispositifs basés sur des principes didactiques reconnus à de nouveaux objets d'enseignement exigeait une phase d'exploration faite 
de tâtonnements et d'expérimentations ( $1^{\text {re }}$ année du projet) nécessitant une étroite collaboration de tous les participants (enseignants, chercheuses universitaires et conseillères pédagogiques ( $\mathrm{CP})$ ). Cette collaboration s'est actualisée par un accompagnement soutenu et régulier qui s'est poursuivi à la $2^{\mathrm{e}}$ année du projet, permettant ainsi la mise au point de dispositifs didactiques à la $1^{\text {re }}$ année et leur expérimentation avec prise de données à la $2^{\mathrm{e}}$ année. Cet article vise à décrire l'accompagnement professionnel et analyser cette collaboration par le regard des conseillères pédagogiques à l'aide d'un questionnaire écrit recueilli au terme de la $2^{\mathrm{e}}$ année du projet. Des pistes pour le développement professionnel des enseignants seront dégagées.

\section{Problématique}

3 Le développement professionnel des enseignants, soit «le processus d'acquisition des savoirs [notamment par la formation continue] qui provoque, par la suite, des changements chez l'enseignant ainsi que des nouveautés sur le plan de sa pratique » (Uwamariya et Mukamurera, 2005 : 142) demeure un enjeu connu dans le monde de l'éducation. D'ailleurs, une large étude américaine a montré qu'au-delà des cinq premières années d'expérience, la très grande majorité des enseignants cessent d'évoluer et de bonifier leurs pratiques pédagogiques (TNTP, 2015). Plusieurs rapports ont également indiqué que les formations offertes dans les milieux scolaires étaient loin d'être optimales (Conseil supérieur de l'éducation, 2014) puisqu'elles ne répondent pas aux critères identifiés par la recherche sur le développement professionnel à savoir que les activités de formation devraient 1- être étendues dans le temps, soit une cinquantaine d'heures sur une durée de plus d'un an (Darling-Hammond et al., 2009; Richard, 2017); 2- être à la base de la construction de bonnes relations entre enseignants pour permettre des discussions riches et ouvertes donnant lieu à un partage d'expertise (Darling-Hammond et al., 2009; Fullan, 2007 ; Richard, 2017) ; 3porter sur un aspect très précis du curriculum, ancré dans les besoins et les visées des écoles (Darling-Hammond et al., 2009) et 4- être étayées par des données probantes, animées par des spécialistes dont l'expertise est reconnue et soutenue par une direction d'établissement faisant preuve de leadership (Richard, 2017). Par ailleurs, les dispositifs didactiques peuvent être considérés comme des vecteurs de développement professionnel puisque l'enseignement représente une activité médiatisée par des outils didactiques. Selon Cèbe et Goigoux $(2007,2012)$ et Goigoux $(2011)$, ces outils doivent être pertinents (exploitant les connaissances issues de la recherche), proches des pratiques habituelles des enseignants, élaborés de manière collaborative et ajustés dans le temps par leur pratique régulière.

Dans le cadre de notre étude, afin de documenter les effets de la mise en œuvre de dispositifs didactiques sur les compétences des élèves en écriture, les enseignants participants devaient non seulement développer des gestes pédagogiques en cohérence avec les principes didactiques retenus, mais également développer des savoirs en grammaire moderne ${ }^{2}$ leur permettant de favoriser et de soutenir les apprentissages de leurs élèves en S-P. L'équipe de recherche devait donc assurer un accompagnement professionnel soutenu afin de leur permettre de s'approprier une posture et des pratiques nouvelles pour lesquelles aucun modèle n'existait, seulement un cadre théorique. De plus, l'équipe a rapidement ressenti le besoin de se confronter au terrain 
pour s'assurer que les dispositifs soient réalistes et fonctionnels. Des échanges réguliers, nourris par l'expertise des $\mathrm{CP}$, ont permis à la recherche collaborative de se fonder sur des besoins partagés par les trois parties.

5 Nous définirons dans la prochaine section les concepts de recherche collaborative, de dispositif didactique et d'accompagnement professionnel. Par la suite, nous décrirons la méthodologie employée pour dégager les perceptions des CP. Les résultats seront ensuite explicités et discutés.

\section{Cadre conceptuel}

6 Selon Desgagné, Bednarz, Lebuis, Poirier et Couture (2001), la recherche collaborative ne porte pas sur les praticiens, mais s'établit avec eux. Elle implique une interinfluence entre la pratique et la recherche et elle prend « le sens d'un échange de services entre des acteurs qui font partie de cultures différentes et qui n'ont pas à répondre aux mêmes finalités " (Desgagné et al., 2001: 39). Dans notre étude, les enseignants se basaient sur un cadre théorique et sur les premières ébauches d'implantation proposées par les chercheuses, pour les expérimenter en classe de concert avec les chercheuses et les CP. Par la suite, les discussions entre tous les acteurs ont permis d'identifier les gestes pédagogiques les plus efficaces et efficients pour la classe. « La recherche collaborative valorise (...) l'intégration des points de vue [des praticiens] dans la production de savoirs aménagée par le chercheur" (Morrissette, 2013: 46). Ainsi, les chercheuses ont bénéficié des savoirs d'expérience des enseignants et des CP participants pour mettre au point les dispositifs didactiques expérimentés afin qu'ils soient mieux ancrés dans la réalité du monde scolaire, donc plus facilement transférables. Les rencontres collectives sur la mise en œuvre d'activités en classe permettaient cette intégration des trois points de vue et le réajustement progressif des dispositifs didactiques initialement proposés.

7 Notre équipe définit le dispositif didactique comme un "ensemble de moyens, matériels ou sémiotiques, fruits d'un travail d'ingénierie a priori mais ajustés à la réalité du milieu, et organisés pour permettre à l'élève de construire des savoirs » (inspiré de Cèbe et Goigoux [2007] et de Weisser [2010]). Dans ce projet, les dispositifs didactiques abordent un contenu précis (ici la S-P), et visent des objectifs également précis (dégager les structures de phrases à partir des outils de la grammaire moderne, dont la notion de phrase et les manipulations syntaxiques, afin de bien les construire et les ponctuer). Les activités proposées aux élèves sont organisées et reliées pour répondre à cet objectif, dans un temps relativement restreint (cf. annexe 1) afin de respecter la réalité quotidienne des classes. On a mis à profit des outils matériels pour les élèves (cahier, corpus de phrases, capsule vidéo, aide-mémoire) et pour l'enseignant (démarche proposée, gestes didactiques, routine, outils numériques (cf. annexe 2).

8 Afin d'éprouver concrètement les dispositifs proposés et de soutenir leur mise en œuvre dans la classe par les enseignants, un accompagnement professionnel a été nécessaire. Aux conditions organisationnelles précédemment évoquées, s'ajoutent des conditions intrinsèques aux enseignants.

9 Selon Shulman et Shulman (2004), l'enseignant doit développer quatre dimensions personnelles de manière simultanée: sa vision, son engagement, ses savoirs et ses pratiques. Autrement dit, les nouveaux savoirs et les nouvelles pratiques développées doivent être en cohérence avec sa vision de l'enseignement, ses valeurs et ses croyances 
au sujet de l'apprentissage pour lui permettre un engagement suffisant dans l'ajustement de ses pratiques et la construction de ses savoirs. De plus, selon Guskey et Yoon (2009), c'est lorsque l'enseignant perçoit l'effet de ses pratiques sur les apprentissages de ses élèves que l'impact sur sa vision est le plus important. Ainsi, pour que l'enseignant en perçoive les effets dans sa classe, les formateurs doivent soutenir l'évolution des quatre dimensions par des modalités de développement professionnel différenciées et par le soutien à l'implantation rapide de nouvelles pratiques. Des rétroactions régulières et formatives soutiennent les apprentissages en ayant lieu dans un contexte ouvert d'apprentissage et en fonction des besoins observés chez les élèves. Enfin, le développement professionnel montre un effet plus grand lorsqu'il est partagé entre collègues (Darling-Hammond et al., 2009).

$10 \mathrm{Au}$ Québec, les $\mathrm{CP}$ jouent un rôle de premier plan dans le développement professionnel des enseignants (Guillemette, Vachon et Guertin, 2019). À ce titre, on leur reconnait un regard d'expert sur les situations de formation (Draelants, 2007). Leur rôle est généralement associé à des interventions hors classe. Quatre situations emblématiques clarifient le travail des $\mathrm{CP}$ : former, conseiller, accompagner et innover (Guillemette et al., 2019). Lors de la phase exploratoire collaborative, les chercheuses ont endossé le rôle d'accompagnement car le besoin de se confronter régulièrement à la réponse des élèves et des enseignants a permis de mieux cerner les enjeux des apprentissages et de mieux réajuster le design des dispositifs. À cette étape du projet de recherche, les CP ont plutôt revêtu un rôle de conseillères, principalement en donnant leur avis sur la conception et la mise en œuvre des dispositifs. Elles ont également partagé la tâche d'accompagnement de l'équipe de chercheuses en participant à l'observation en classe et à la rétroaction fournie aux enseignants, ce qu'elles font peu habituellement.

\section{Méthodologie}

Cet article s'intéresse au point de vue des $\mathrm{CP}$ sur l'accompagnement offert dans le cadre de notre recherche.

\section{Participants}

12 Rappelons que cette étude a développé des dispositifs didactiques pour enseigner la S-P au $3^{\mathrm{e}}$ cycle du primaire (10-11 ans) et au $1^{\mathrm{er}}$ cycle du secondaire (12-13 ans) dans des milieux défavorisés au Québec où les élèves éprouvent plus de difficultés à réussir les examens ministériels en lecture et en écriture et représentent donc une population à risque (Desrosiers et Tétrault, 2012). Ainsi, plusieurs dispositifs didactiques ont été conçus et ajustés en collaboration avec les cinq membres de l'équipe de recherche, quatre $\mathrm{CP}$ et treize enseignants. Les $\mathrm{CP}$ et les enseignants, approchés en raison de leur désir d'apprendre des activités innovantes en S-P, étaient volontaires pour participer à l'étude et leurs directions respectives soutenaient leur implication. Les participants étaient répartis géographiquement en trois groupes dont deux incluaient des $\mathrm{CP}$ : deux $\mathrm{CP}$, Danielle ${ }^{3}$ et Chantale, dans un milieu mixte francophone et plurilingue et deux CP, Josianne et Marie-Claude, dans un milieu unilingue francophone. 


\section{Contexte de l'étude}

13 Les chercheuses se sont appuyées sur la littérature scientifique (Darling-Hammond et al, 2009; Richard, 2018; Shulman et Shulman, 2004; TNTP, 2015) pour offrir aux enseignants un accompagnement structuré autour de trois modalités : 1-des rencontres collectives mensuelles sur une période de 18 mois au cours desquelles les activités étaient explicitées et où les enseignants échangeaient sur leur expérience, 2- des observations bimensuelles en classe suivies de rétroactions personnalisées aux enseignants sur leur métalangage et leurs pratiques plus fines (par ex. : leur manière d'utiliser les manipulations syntaxiques ou de questionner les élèves). Ces observations ont été réalisées par les chercheuses accompagnées parfois des CP dans le but de développer les pratiques didactiques attendues, mais aussi de mieux comprendre la mise en œuvre des dispositifs expérimentés et 3- du matériel pour soutenir l'implantation des dispositifs, ajusté et offert à tous les participants au cours de l'expérimentation (cf. annexe 2). De multiples allers-retours entre la conception, l'ajustement et l'expérimentation des dispositifs ont été nécessaires pour obtenir la séquence finale qui a été mise en œuvre et mesurée dans des textes d'élèves lors de l'année scolaire suivante. Les résultats chez les élèves en écriture feront l'objet d'autres publications.

\section{Outils de collecte et d'analyse}

$14 \mathrm{Au}$ terme de l'expérimentation, un questionnaire d'enquête à réponse ouverte (Gaudreau, 2011) a été distribué aux CP sur différents thèmes liés au développement professionnel (cf. annexe 3). L'analyse du questionnaire écrit auquel trois CP ont répondu (Danielle, Chantale et Josianne - Marie-Claude a été relancée deux fois, mais n'a pas remis son questionnaire) a fait l'objet d'une analyse thématique basée sur les éléments-clés liés aux composantes efficaces du développement professionnel.

\section{Résultats : Analyse des questionnaires complétés par les CP}

15 Dans un premier temps, les trois CP évoquent que le métalangage lié à la grammaire moderne représente un apprentissage signifiant pour les enseignants. Les manipulations syntaxiques ainsi que les concepts de phrase graphique et phrase syntaxique ont constitué également des apprentissages importants pour ces derniers. En ce qui concerne les apprentissages liés aux pratiques didactiques, elles remarquent que la démarche inductive, le questionnement et la discussion-négociation sollicités par les activités ont permis aux enseignants de donner davantage la parole aux élèves, ce qui a modifié leur vision de ces pratiques. Quant à leurs propres apprentissages, Chantal et Josianne mentionnent que les manipulations et les contextes utilisés dans les exercices permettent de mieux discriminer les compléments de phrase des compléments indirects de même que les procédés de combinaison de juxtaposition, de coordination et de subordination. Quant aux apprentissages didactiques, les trois CP disent mieux comprendre comment enseigner la S-P autrement que par des leçons de grammaire isolées de l'écriture de textes. Elles saisissent mieux la nécessité d'établir une routine tant pour les enseignants que pour les élèves afin de baliser le pilotage de 
l'activité. Enfin, toutes les trois disent avoir affiné leur capacité à questionner les enseignants et à vulgariser les concepts relatifs à la S-P.

Dans un deuxième temps, les $\mathrm{CP}$ ont été invitées à se prononcer sur les modalités d'accompagnement. Selon Danielle, «l'avantage des rencontres collectives, c'est de permettre aux enseignants de faire part des difficultés rencontrées et d'échanger sur des moyens possibles de les surmonter ». Josianne a d'ailleurs observé une évolution dans les échanges au fur et à mesure que la confiance mutuelle s'installait. Ce partage $a$, selon les CP, un effet d'émulation pour certains enseignants. Comme le souligne Chantale, « les rencontres collectives leur ont permis de voir d'autres enseignants qui réussissent à changer leur enseignement de la grammaire, à proposer des activités différentes pour permettre aux élèves de mieux apprendre les concepts ». Les trois $\mathrm{CP}$ mentionnent que ces rencontres s'avèrent rassurantes pour les enseignants, car elles leur permettent de valider leur compréhension des activités, de s'approprier la démarche et les modalités d'application des activités à venir, d'ajuster leur vision de l'apprentissage et de l'enseignement. Par contre, Danielle aurait souhaité offrir un approfondissement sur des concepts grammaticaux sollicités en S-P pour permettre aux enseignants de développer une meilleure assurance dans l'animation des activités en classe.

17 En ce qui concerne le matériel offert, les trois $\mathrm{CP}$ indiquent qu'il facilite la mise en œuvre des activités en raison du temps que cette planification nécessite. Elles ont apprécié la variété des activités proposées, ce qui permet aux enseignants de faire des choix selon les besoins des élèves. Enfin, elles jugent que le guide de l'enseignant fourni de même que les capsules vidéo sont très encadrants pour ces derniers, car cela leur offre un modèle sur lequel s'appuyer. Josianne mentionne également qu'elle a apprécié le fait que les chercheuses aient ajusté les activités proposées entre la fin de l'année de recherche collaborative et l'année d'expérimentation des dispositifs en tenant compte des commentaires reçus et des liens avec le curriculum.

Quant aux observations et aux rétroactions en classe, les trois CP affirment que cela a surtout contribué à la mise en action réelle des dispositifs proposés. De plus, cela leur a donné l'occasion d'observer la réalisation des activités - ce qui s'avère assez rare dans le cadre de leur travail. La présence en classe de $\mathrm{CP}$ et de chercheuses génère un stress important chez les enseignants, mais cette présence est tout autant sécurisante et elles aiment être sollicitées à titre d'expertes pour répondre à des questions sur le vif. Pour Chantale, «la présence en classe me permet de les rassurer, d'entrer dans leur planification des apprentissages et d'avoir des discussions sur leurs gestes pédagogiques, leurs questions et les problèmes grammaticaux rencontrés. Autrement, elles ne m'auraient pas contactée pour cela ».

19 Enfin, dans un troisième temps, les $\mathrm{CP}$ ont discuté de leur rôle au sein du projet de recherche. Elles disent avoir assumé un rôle de guide, de soutien, de facilitatrice, principalement dans le cadre des observations où elles ont parfois fait du coenseignement. Elles évoquent que leur présence était perçue comme sécurisante pour les enseignants puisqu'elles étaient en mesure de faire des liens avec le curriculum et d'agir à titre de référence au moment opportun. Ce rôle a accru, selon elles, la relation de confiance qu'elles entretiennent avec les enseignants. Leur présence physique à l'école a permis aux enseignants de poser plus de questions, mais aussi d'ouvrir des discussions avec les autres membres de l'équipe école ne participant pas au projet à propos de l'enseignement de l'écriture et de la grammaire. Elles ont vécu leur rôle 
comme celui d'un chainon important entre les enseignants, les chercheuses et les directions d'école. Danielle et Chantale auraient souhaité participer à l'élaboration des rencontres de recherche et du matériel expérimenté conjointement avec les chercheuses car, selon elles, cela aurait contribué à leur propre formation. Josianne, quant à elle, en a eu l'occasion dans son terrain de recherche.

Dans une optique de maintien et de transfert des pratiques, elles croient que leur rôle sera de maintenir les échanges sur les pratiques par des rencontres collectives et individuelles. Elles jugent que certains enseignants auront toujours besoin de soutien, mais elles doutent qu'ils accepteront de l'aide une fois le projet de recherche terminé. En effet, selon les trois CP, le contexte de la recherche "crée " une occasion de développement professionnel. Or, le contexte organisationnel dans lequel elles se trouvent ne leur permet pas souvent de réaliser des suivis: elles se sentent dépendantes des priorités de leur commission scolaire et des budgets qui y sont associés. Danielle et Josianne misent ainsi sur une forme de " contamination positive » des enseignants participants pour une diffusion des pratiques dans leur école.

Les trois $\mathrm{CP}$ qui ont répondu au questionnaire constatent donc des apprentissages chez les enseignants tant sur le plan des pratiques que des savoirs. Parce qu'elles répondent à des intentions différentes, les CP jugent les trois modalités d'accompagnement nécessaires et complémentaires. Elles considèrent avoir rempli un rôle de conseil, de guide, de soutien même si certaines auraient souhaité accompagner davantage au sens où Guillemette et al. (2019: 43) décrivent ces rôles, soit le fait "d'accompagner le personnel scolaire dans un contexte de collaboration ou de mobilisation pour mieux soutenir l'amélioration des pratiques éducatives, pédagogiques ou didactiques ».

\section{Discussion}

22 La dimension exploratoire de cette recherche a demandé la collaboration étroite de trois acteurs clés du développement professionnel: les CP, les enseignants et les chercheuses. Les rôles traditionnels de ces intervenants ont été plus souples que dans un autre type de recherche : les chercheuses n'ont pas été les seules conceptrices des dispositifs, les CP ne les ont pas que validés et les enseignants ne les ont pas qu'expérimentés. Ainsi, c'est par une réelle collaboration qu'ont pu se développer les dispositifs de la séquence didactique finale, une collaboration essentielle pour leur acceptabilité par les intervenants du milieu scolaire, une conciliation, en somme, entre le « souhaitable » des chercheuses et le « réalisable » des enseignants (Goigoux et Cèbe, $2009 ; 2011)$.

23 Le contexte de la recherche, par l'implication volontaire des enseignants, a facilité la mise en œuvre de modalités d'accompagnement, telles que l'observation en classe suivie d'une rétroaction. Bien que reconnue efficace comme dispositif de développement professionnel (TNTP, 2015), elle représente sans doute la modalité la plus déstabilisante pour les enseignants. Ces derniers, dits "précurseurs", sentent qu'ils ont toujours aimé se développer, tenter de nouvelles pratiques, apprendre. Cet engagement s'avère plus fort que le stress engendré par la présence de chercheuses et de $\mathrm{CP}$ en classe. Il semble qu'il faille ici trouver l'équilibre le plus juste (différent pour chaque enseignant) entre une présence anxiogène qui pousse à se dépasser et une présence rassurante qui guide dans les moments d'incertitude. Les trois CP ont 
explicitement nommé la relation de confiance à établir avec les enseignants, relation renforcée par cette présence rapprochée.

L'objet de développement à proprement parler représente une autre explication à l'ouverture à l'observation. Généralement, lorsque les CP vont en classe, c'est bien souvent pour modéliser une pratique attendue. On tente de montrer aux enseignants un mode d'emploi à transposer, voire à imiter selon une vision applicationniste (Goigoux et Cèbe, 2009). Alors que la présence d'une tierce personne en classe serait généralement associée à une évaluation de la performance selon des critères attendus, le sentiment de contribuer à une innovation pédagogique permettrait une plus grande ouverture des enseignants et ce, malgré l'inconfort à être observé (Cèbe et Goigoux, 2007, 2012 ; Goigoux, 2011 ; Giguère et al., 2018).

Des différences individuelles entre les CP ont été observées dans l'analyse des réponses au questionnaire. Danielle et Chantale auraient souhaité un rôle plus actif dans la planification et l'animation des rencontres collectives, et dans la formation en grammaire offerte aux enseignants. D'une part, cette place n'a pas pu être offerte, car les chercheuses créaient les dispositifs et avaient besoin d'un contact direct avec le terrain pour observer le déroulement des activités et bénéficier d'un accès aux conceptions des élèves et des enseignants. D'autre part, la disponibilité et la flexibilité des horaires des membres de l'équipe de recherche a permis cet accompagnement rapproché, ce que les $\mathrm{CP}$ n'auraient pas pu offrir, étant donné les contraintes organisationnelles de leur travail, leurs nombreux dossiers et engagements fixés longtemps à l'avance.

Enfin, les CP ont partagé leur expertise relative aux conditions de maintien et de transfert des dispositifs, un enjeu important de valorisation des connaissances issues de la recherche dans les milieux de pratique. Selon leurs réponses au questionnaire, l'accompagnement soutenu, à la fois individuel et collectif, permet un maintien des pratiques, mais également un engouement pour l'objet de développement dans les milieux de pratique, ce qui représente un levier pour les CP dans l'accompagnement de nouveaux enseignants n'ayant pas participé au projet de recherche.

Ces résultats ne sont pas généralisables en raison du faible nombre de participants et $\mathrm{du}$ caractère volontaire de leur engagement. Il est également difficilement envisageable de généraliser ce genre de collaboration au Québec en raison des difficultés de recrutement de participants volontaires. En revanche, une telle collaboration pourrait être possible sans l'apport des chercheuses à la condition que les milieux scolaires (écoles et commissions scolaires) dégagent du temps de qualité et des budgets substantiels pour permettre à des $\mathrm{CP}$ d'endosser un rôle accru d'innovation dans lequel des rencontres collectives régulières, des suivis réguliers en classe et un partage de matériel pourraient être offerts pour répondre aux besoins des écoles. Enfin, une évaluation des résultats devrait faire partie de cet accompagnement.

\section{Conclusion}

La recherche que l'équipe de cinq chercheuses a menée en collaboration avec des enseignants et des CP s'appuie d'abord sur les principes de la recherche collaborative afin que chaque participant profite d'un échange pour répondre à des finalités différentes : selon les $\mathrm{CP}$, les enseignants ont réalisé des apprentissages en S-P et dans leur enseignement, elles évoquent elles-mêmes des apprentissages sur le 
questionnement et l'observation en classe, et estiment que les chercheuses ont structuré une séquence d'activités basées sur les principes didactiques et sur les besoins des élèves et des enseignants (métalangage, questionnement, interactions).

Le contexte d'une recherche semble faciliter la mise en œuvre rapide de dispositifs innovants par les enseignants participants, comme une obligation à développer leurs pratiques dans un délai plus court que ce qu'ils auraient fait dans le cadre d'une formation plus régulière. Les enseignants comme les $\mathrm{CP}$ en ont vu les bénéfices entre autres dans la fluidité des pratiques, dans l'affirmation des savoirs, mais également dans la transformation de la vision de l'enseignement de la syntaxe et de la ponctuation, dont l'enseignement est problématique dans plusieurs milieux scolaires (Boivin et Pinsonneault, 2012). Toutefois, des questions soulevées par les $\mathrm{CP}$ de l'étude à propos de l'ouverture de la classe se posent toujours: comment développer les différentes dimensions du développement professionnel dans le contexte des contraintes organisationnelles dans les commissions scolaires, et comment maintenir les pratiques dans les années à venir lorsque le soutien se retire?

\section{BIBLIOGRAPHIE}

Boivin, M.-C. (2014). Quand les élèves « font de la grammaire » en classe : analyse d'interventions métalinguistiques d'élèves du secondaire. Dans Garcia-Debanc, C., V. Paolacci et M.-C. Boivin (dir.), Repères, L'étude de la langue : Des curricula aux pratiques observées, 49, 131-145. http:// reperes.revues.org/717.

Boivin, M.-C. et Pinsonneault, R. (2018). Les erreurs de syntaxe, d'orthographe grammaticale et d'orthographe lexicale des élèves québécois en contexte de production écrite. Revue canadienne de linguistique appliquée, 21(1), 43-70. https://journals.lib.unb.ca/index.php/CJAL/article/view/ 25121.

Brissaud C., et Cogis, D. (2011). Comment enseigner l'orthographe aujourd'hui ? Paris : Hatier.

Cèbe, S. et Goigoux, R. (2007). Concevoir un instrument didactique pour améliorer l'enseignement de la compréhension de textes. Repères, recherches en didactique du français langue maternelle, 35, 185-208. DOI : https://doi.org/10.3406/reper.2007.2756.

Cèbe, S. et Goigoux, R. (2012). Comprendre et raconter : de l'inventaire des compétences aux pratiques d'enseignement. Le français aujourd'hui, 179(4), 21-36.

Conseil supérieur de l'éducation (2014). Le développement professionnel, un enrichissement pour toute la profession enseignante. Québec, Gouvernement du Québec.

Chartrand, S.-G. (2011). Grammaire pédagogique du français d'aujourd'hui (2e éd.) Montréal : Gaficor. Chartrand, S.-G. (2009). Enseigner la grammaire autrement. Québec français, Hors-série, 13-15.

Darling-Hammond, L., Wei, R. C., Andree, A., Richardson, N., et Orphanos, S. (2009). Professional learning in the learning profession. Washington, DC : National Staff Development Council. 
Desgagné, S., Bednarz, N., Lebuis, P., Poirier, L. et Couture, C. (2001). L'approche collaborative de recherche en éducation : un rapport nouveau à établir entre recherche et formation. Revue des sciences de l'éducation, 27(1), 33-64. https://doi.org/10.7202/000305ar.

Desrosiers, H. et Tétrault, K. (2012). Les facteurs liés à la réussite aux épreuves obligatoires de français en sixième année du primaire : un tour d'horizon. Étude longitudinale du développement des enfants du Québec (ÉLDEQ 1998-2010) - De la naissance à 12 ans. Québec : Institut de la statistique du Québec, 7(1), 1-40.

Draelants, H. (2007). Entre le pair et l'expert, trouver la distance qui convient : une question de légitimation pour le conseiller pédagogique. Recherches sociologiques et anthropologiques, 38 (1), 163-182.

Fullan, M. (2007). The New Meaning of Educational Change (4th ed.). New York : Teachers college Press.

Gaudreau, L. (2011). Guide pratique pour créer et évaluer une recherche scientifique en éducation. Montréal : Guérin.

Giguère, M.-H., Nadeau, M., Fisher, C., Arseneau, R. et Quevillon Lacasse, C. (2018). Dialogue enseignants-chercheurs dans un accompagnement différencié pour développer le savoir-faire des élèves en syntaxe et en ponctuation. Dans Briquet-Duhazé, S. et Turcotte, C., La diffusion et l'appropriation des résultats de recherche en lecture/écriture dans les milieux de la pratique. Londres : ISTE.

Goigoux, R. et Cèbe, S. (2009). Un autre rapport entre recherche, pratique et formation. Les instruments didactiques comme vecteur de transformation des pratiques des enseignants confrontés aux difficultés d'apprentissage des éleves. Conférence invitée en clôture du colloque du réseau international de Recherche en Éducation et Formation (REF), Université de Nantes, 19 juin 2009.

Goigoux, R. et Cèbe, S. (2011). Un autre rapport entre recherche, pratique et formation. Les instruments didactiques comme vecteur de transformation des pratiques des enseignants confrontés aux difficultés d'apprentissage des élèves. Conférence de clôture du 9ème colloque international de Recherche en éducation et formation (REF). Nantes, 2009. https://halshs.archivesouvertes.fr/halshs-00936348/document.

Goigoux, R. (2011). Une pédagogie éclectique au service des élèves qui ont le plus besoin de l'école. La nouvelle revue de l'adaptation et de la scolarisation, 52, 22-30.

Guillemette, S., Vachon, I. et Guertin, D. (2019). Référentiel de l'agir compétent en conseillance pédagogique en soutien à la réussite des élèves. Montréal : Éditions JFD inc.

Guskey, T. R., et Yoon, K. S. (2009). What Works in Professional Development ? Phi Delta Kappan, 90(7), 495-500. https://doi.org/10.1177/003172170909000709.

Jaffré, J.-P. (2014) À quoi sert la ponctuation ? Le français aujourd'hui, 4(187), 129-135. https:// www.cairn.info/revue-le-francais-aujourd-hui-2014-4-page-129.htm.

Morrissette, J. (2013). Recherche-action et recherche collaborative : quel rapport aux savoirs et à la production de savoirs? Nouvelles pratiques sociales, 25 (2), 35-49. https://doi.org/ 10.7202/1020820ar.

Nadeau, M., et Fisher, C. (2009). Faut-il des connaissances explicites en grammaire pour réussir les accords en français écrit? Résultats d'élève de 6e année du primaire. Pratiques d'enseignement grammatical. Québec: PUL, 209-231. 
Nadeau, M. et Fisher, C. (2011). Les connaissances implicites et explicites en grammaire : quelle importance pour l'enseignement? Quelles conséquences? Bellaterra Journal of Teaching \& Learning Language \& Literature. 4.4 (Nov-Dec), 1-31.

Nadeau, M. et Fisher, C. (2014). Expérimentation de pratiques innovantes, la dictée 0 faute et la phrase dictée du jour, et étude de leur impact sur la compétence orthographique des élèves en production de texte. Rapport de recherche. FRQSC, Programme Actions concertées.

Paolacci, V. et Garcia-Debanc, C. (2003). Quel enseignement de la ponctuation (et autres marques d'organisation textuelle) en formation initiale des enseignants ?. Repères, ${ }^{\circ}$ 28, p. 93-116.

Paolacci, V. et Rossi-Gensane, N. (2014). Ponctuation et écrits d'élèves : Une conception différente de la phrase pour enseigner la ponctuation autrement. Le français aujourd'hui, 4(187), 115-125.

Richard, M. (2017). Quels sont les modèles de formation continue les plus efficaces pour l'enseignement de la lecture et de l'écriture chez les élèves du préscolaire, du primaire et du secondaire ? Une synthèse des connaissances. Rapport de recherche FRQSC. [En ligne].

Shulman, L., et Shulman, J. (2004). How and what teachers learn : a shifting perspective. Journal of Curriculum Studies, 36(2), 257-271.

The New Teacher Project - TNTP (2015). The Mirage. Confronting the Hard Truth About Our Quest for Teacher Development. https://tntp.org/ (consulté le 17 novembre 2015).

Uwamariya, A., et Mukamurera, J. (2005). Le concept de « développement professionnel » en enseignement : approches théoriques. Revue des sciences de l'éducation, 31(1), 133-155. http:// id.erudit.org/iderudit/012361ar.

Weisser, M. (2010). Dispositif didactique ? Dispositif pédagogique ? Situations d'apprentissage !, Questions Vives, vol. $4 \mathrm{n}^{\circ}$ 13, mis en ligne le 26 janvier 2011, consulté le 22 août 2019. URL : http:// journals.openedition.org/questionsvives/271.

\section{ANNEXES}

\section{Annexe 1 - Séquence didactique du projet}

\begin{tabular}{|l|l|}
\hline Dispositifs & Prévoir de 30 à 40 min par activité \\
\hline $\begin{array}{l}\text { Notions de phrase syntaxique et } \\
\text { phrase graphique }\end{array}$ & 3 à 4 « périodes » avec manipulation de « cartons » \\
\hline Ajout de ponctuation & $\begin{array}{l}6 \text { activités d'ajout de ponctuation dans un texte sans majuscules ni } \\
\text { points. }\end{array}$ \\
\hline Combinaison de phrases & $\begin{array}{l}10 \text { activités où plusieurs phrases simples doivent être jointes en } \\
\text { une seule phrase graphique par divers procédés. }\end{array}$ \\
\hline
\end{tabular}

\section{Annexe 2: Matériel offert aux enseignants}

Cahier de l'élève (un pour le primaire et un pour le secondaire) regroupant tous les exercices suivis d'une section «À retenir »; 
Guide de l'enseignant (pour le primaire et pour le secondaire) présentant des solutionnaires, des guides d'animation des différents dispositifs, des tableaux illustrant des possibilités grammaticales pouvant survenir en classe lors des discussions avec les élèves ;

Activités « clé en main » sur les concepts de phrases graphiques et phrases syntaxiques incluant les cartons à manipuler par les élèves ;

Capsules vidéo (une pour le primaire et une pour le secondaire) montrant un modelage du dispositif « combinaison de phrases »;

Pages pour le tableau numérique interactif (pour le primaire et pour le secondaire) servant à l'animation des échanges avec les élèves.

\section{Annexe 3 : Questionnaire individuel aux CP}

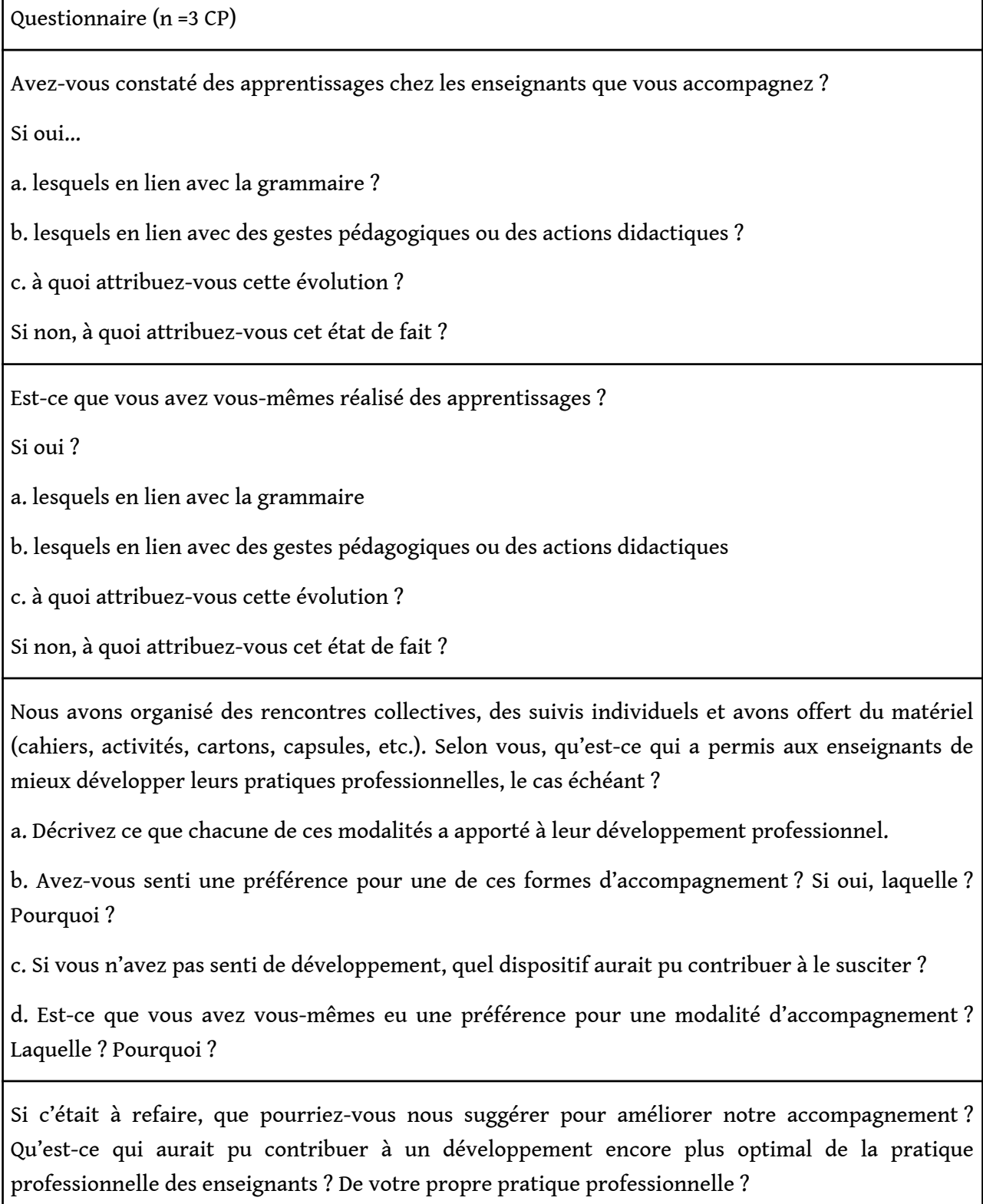

Nous avons organisé des rencontres collectives, des suivis individuels et avons offert du matériel (cahiers, activités, cartons, capsules, etc.). Selon vous, qu'est-ce qui a permis aux enseignants de mieux développer leurs pratiques professionnelles, le cas échéant?

a. Décrivez ce que chacune de ces modalités a apporté à leur développement professionnel.

b. Avez-vous senti une préférence pour une de ces formes d'accompagnement? Si oui, laquelle? Pourquoi?

c. Si vous n'avez pas senti de développement, quel dispositif aurait pu contribuer à le susciter?

d. Est-ce que vous avez vous-mêmes eu une préférence pour une modalité d'accompagnement? Laquelle? Pourquoi?

Si c'était à refaire, que pourriez-vous nous suggérer pour améliorer notre accompagnement? Qu'est-ce qui aurait pu contribuer à un développement encore plus optimal de la pratique professionnelle des enseignants? De votre propre pratique professionnelle? 
Le projet se termine dans quelques semaines. Envisagez-vous un maintien des pratiques dans les classes expérimentales? Les enseignants auront-ils besoin de soutien? Si oui, lequel ? Si non, à quoi attribuez-vous le fait que ce ne soit pas nécessaire?

Envisagez-vous un transfert des pratiques dans d'autres classes ou d'autres écoles? Si oui, comment cette diffusion devrait être organisée? De quoi auriez-vous besoin pour mener un tel accompagnement? Si non, à quoi attribuez-vous le fait de ne pas donner suite à ce projet ?

Quel rôle le projet vous a-t-il permis de prendre dans le cadre de vos fonctions de CP ?

Est-ce que ce rôle vous a permis de travailler différemment? Si oui, comment ? Pourquoi ?

\section{NOTES}

1. Recherche financée par le FRQSC-MEES (Fonds de recherche québécois - société et culture Ministère de l'éducation et de l'enseignement supérieur): Expérimentation de dispositifs didactiques en syntaxe et en ponctuation «à la manière » des dictées métacognitives et interactives (2017-LC-198593).

2. Selon Chartrand (2011), la grammaire dite moderne ou nouvelle se veut une description du «fonctionnement de la langue et des textes en présentant des informations sur [...] les outils pour travailler la langue (le modèle de la phrase $\mathrm{P}$ et les manipulations syntaxiques), les structures syntaxiques de la phrases, les principales règles d'orthographe et de ponctuation, ainsi que l'histoire et le fonctionnement du lexique » (avant-propos, III).

3. Prénoms fictifs pour préserver l'anonymat.

\section{RÉSUMÉS}

L'étude présentée dans cet article s'est déroulée en deux temps : une démarche exploratoire la première année, suivie d'une expérimentation par protocole quasi-expérimental l'année suivante. Visant à développer et à expérimenter des dispositifs didactiques pour améliorer la syntaxe et la ponctuation en écriture chez des élèves de 10 à 14 ans, l'équipe de recherche était composée de chercheuses universitaires, d'étudiantes doctorale et postdoctorale, de conseillères pédagogiques et d'enseignants du primaire et du secondaire au Québec. Tous ces membres ont joué différents rôles tout au long de l'étude. Cet article vise à présenter les résultats de l'analyse d'un questionnaire d'enquête auprès des conseillères pédagogiques dans lequel les modalités d'accompagnement professionnel ont été discutées. Le point de vue des conseillères pédagogiques montre que la recherche collaborative permet de documenter l'efficacité d'un triple accompagnement (rencontres collectives mensuelles, observations en classe avec rétroaction personnelle, matériel offert) et de partager des rôles traditionnellement plus cloisonnés.

The study reported in this article was carried out in two phases: the first year followed an exploratory approach for the creation of teaching devices, and the second year tested these devices through a quasi-experimental protocol. The research team, interested with developing 
and experimenting with teaching devices to improve syntax and punctuation in writing for students aged 10 to 14, was composed of university researchers, doctoral and postdoctoral students, teacher consultants, as well as elementary and secondary teachers in Quebec. All of these members played different roles throughout the study. This article presents the results of a survey questionnaire submitted to the teacher consultants in which the means to support teacher professional development (TPD) were discussed. The stances from teacher consultants show that this collaborative research made it possible to document the effectiveness of a triple TPD support (monthly group meetings, classroom observations with personal feedback, and provided material), while allowing for a shift of traditionally compartmentalized roles in research.

\section{INDEX}

Mots-clés : développement professionnel, accompagnement, conseillères pédagogiques, séquence didactique, syntaxe et ponctuation

Keywords : teacher professional development, support, teacher consultants, teaching device, syntax and punctuation

\section{AUTEURS}

\section{MARIE-HÉLÈNE GIGUÈRE}

UQAM

Marie-Hélène Giguère est professeure au département d'éducation et formation spécialisées de l'UQAM depuis 2015. Elle a été enseignante de français au secondaire, puis conseillère pédagogique avant de terminer son doctorat sur un dispositif de développement professionnel portant sur la grammaire actuelle et son enseignement.

giguere.marie-helene[at]uqam.ca

\section{MARIE NADEAU}

UQAM

Professeure au département de didactique des langues de l'UQAM, Marie Nadeau se consacre depuis plus de 20 ans à la recherche en didactique de l'orthographe et de la grammaire. Elle est actuellement la chercheure principale d'un projet sur l'enseignement de la syntaxe et de la ponctuation par des activités innovantes. nadeau.marie[at]uqam.ca

\section{CAROLE FISHER}

UQAC

Après plus de vingt ans comme professeure de didactique du français à l'UQAC, Carole Fisher est maintenant professeure associée à cette même université où elle poursuit des activités de recherche en didactique de l'orthographe et de la grammaire et en didactique de l'oral. c.fisher[at]uqac.ca

\section{ROSIANNE ARSENEAU}

UQAM

Rosianne Arseneau est stagiaire postdoctorale au Département de didactique des langues de l'UQAM et consultante à l'évaluation en français langue seconde au MEES. Elle a été enseignante de français au secondaire avant de terminer son doctorat sur l'enseignement de la phrase 
subordonnée relative.

arseneau.rosianne[at]uqam.ca

\section{CLAUDE QUEVILLON LACASSE}

UQAM

Claude Quevillon Lacasse est doctorante en éducation et chargée de cours au département de didactique des langues de l'UQAM, ainsi que collaboratrice au MEES. Elle s'intéresse, pour son doctorat, à la didactique comparée de la grammaire en langue maternelle et en langue seconde. quevillon_lacasse.claude[at]uqam.ca 